\title{
Continuous Renal Replacement Therapy-Related Strategies to Avoid Colistin Toxicity: A Clinically Orientated Review
}

\author{
Patrick M. Honoréa Rita Jacobs ${ }^{a}$ Olivier Joannes-Boyau ${ }^{d}$ Stijn Lochy ${ }^{a}$ \\ Willem Boer ${ }^{c}$ Elisabeth De Waele ${ }^{a}$ Viola Van Gorp ${ }^{a} \quad$ Jouke De Regt ${ }^{a}$ \\ Vincent Collin ${ }^{b}$ Herbert D. Spapen ${ }^{a}$ \\ a Department of Intensive Care Medicine, Universitair Ziekenhuis Brussel, Vrije Universiteit Brussel, and \\ ${ }^{b}$ Clinique de L'Europe St. Michel, Brussels, and 'Department of Anesthesiology and Critical Care Medicine,

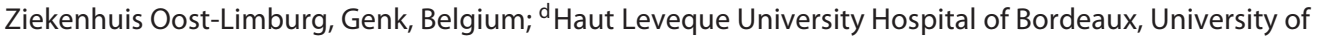 \\ Bordeaux 2, Pessac, France
}

\section{Key Words}

Acute kidney injury · Colistin · Colistin dosing .

Continuous renal replacement therapy - Dialysis .

Nephrotoxicity $\cdot$ Renal replacement therapy

\begin{abstract}
Polymyxins are 'old' antimicrobials which were abandoned for almost 30 years because of significant renal and neurological toxicity. However, the alarming rise in multiresistant Gram-negative bacterial infections worldwide has revived interest in these 'forgotten' agents. Colistin (polymyxin E) is one of the main antibiotics of this class. It is most often administered as the prodrug colistimethate sodium. Doses for treatment of systemic infections in adults range between 3 and 9 million IU per day. Colistin is increasingly used to treat pneumonia and bacteremia in critically ill patients. During their intensive care unit stay, many of these patients will need continuous renal replacement therapy (CRRT) because of acute kidney injury or an unstable hemodynamic condition. Based on recent pharmacological data and our own ex-
\end{abstract}

perience, we postulate that patients undergoing CRRT may receive substantially higher doses of colistin (i.e. a high loading dose, followed by a maintenance dose of up to 4.5 million IU t.i.d.). Treatment can be continued for a prolonged time period without increasing toxicity. CRRT counteracts colistin accumulation because the drug is continuously filtered and also significantly adsorbed in the bulk of the dialysis membrane. Implementing such a 'CRRT rescue' therapy does require the strict use of highly adsorptive dialysis membranes in association with citrate anticoagulation to increase membrane performance.

(c) 2014 S. Karger AG, Basel

\section{Introduction}

After being abandoned in the 1980s because of the high incidence of nephro- and neurotoxicity, the polymyxin antibiotics have been recalled into service as a last-resort treatment of recently emerging multidrugresistant Gram-negative infections [1]. Colistin (poly-

\section{KARGER}

E-Mail karger@karger.com

www.karger.com/bpu
(C) 2014 S. Karger AG, Base

0253-5068/14/0374-0291\$39.50/0
Prof. Patrick M. Honoré, MD, PhD, FCCM

Department of Intensive Care Medicine, University Hospital Brussels Vrije Universiteit Brussel

Laarbeeklaan 101, BE-1090 Brussels (Belgium)

E-Mail Patrick.Honore@uzbrussel.be 
myxin E), in particular, is predominantly and increasingly used to treat nosocomial pneumonia and bacteremia caused by Acinetobacter baumannii, Pseudomonas aeruginosa and Klebsiella pneumoniae.

Pharmacokinetic (PK) and pharmacodynamic (PD) characteristics of colistin are poorly documented. Colistin is mostly administered as the prodrug colistimethate sodium, which undergoes spontaneous hydrolysis to form active colistin [2]. Doses used for systemic infections in adults range between 3 and 9 million IU per day. However, recent PK studies indicate that maximal colistin concentrations achieved following administration of currently recommended doses are low [3]. Combination antimicrobial therapy (e.g. with a carbapenem) is proposed to maximize antimicrobial efficacy, but this approach has no proven clinical advantage over monotherapy [4]. Higher colistin dosing is another option but at the cost of an increased risk for acute kidney injury (AKI). Either a high daily dose [5] or the total cumulative dose [6] of colistin are considered to be potentially predictive for AKI.

Continuous renal replacement therapy (CRRT) has become a standard adjuvant therapy in the intensive care unit (ICU) setting. Apart from commonly accepted indications (AKI of different origin, hemodynamic instability without AKI or fluid overload, for example), CRRT could also be used as a 'supporting' therapy to allow adequate dosing of an antibiotic whilst limiting some or all of its known toxicities. Regarding colistin treatment, CRRT would thus be an elegant method to reconcile optimal efficacy and safety issues when high doses are used for an extended period of time. Inhaled colistin is also another valuable option for resistant Gram-negative infections [1]; the doses are typically 1.5 million IU t.i.d. by the aerosol route [1]. The association of inhaled colistin will not impact the plasma level of colistin as no resorption will occur at the bronchial level [7].

\section{Basic Pharmacological Characteristics of Colistin}

The molecular weight of colistin is about $1,748 \mathrm{Da}$ and thus relatively small. Regarding protein binding, the percentage is about $55 \%$ and thus enabling his removal by CRRT. The distribution volume was about $0.09-0.341 / \mathrm{kg}$, which enables further removal by CRRT. Finally, colistin is a hydrophilic substance and is therefore able to be cleared by water-soluble removal like CRRT $[1,3,7]$.

\section{Colistin Toxicity}

Detecting colistin toxicity at the bedside is cumbersome, in particular when patients are mechanically ventilated. Colistin neurotoxicity should be suspected in case of seizures, prolonged coma or when a previously conscious patient suddenly fails to trigger the ventilator or develops unexplained respiratory muscle paralysis $[1,8$, 9]. A more commonly observed adverse effect following colistin administration is the development of nephrotoxicity. Risk factors for this complication include older age, preexisting renal dysfunction, hypoalbuminemia and concomitant use of intrinsically nephrotoxic drugs (e.g. nonsteroidal anti-inflammatory agents, aminoglycosides or vancomycin). Measuring colistin serum levels is difficult, labor intensive and only feasible in highly specialized laboratories. Moreover, any relationship between a distinct serum concentration and the occurrence of toxicity has not been demonstrated.

\section{Pathophysiology of Renal Toxicity}

The mechanisms of renal toxicity remain a matter of debate. Some reports show a 4 -fold increased risk of nephrotoxicity for patients receiving colistin for $>14$ days, which suggests that toxicity is related to the total dose and duration of therapy. Nephrotoxicity does not appear to be associated with the dose per day $(\mathrm{mg} / \mathrm{kg} / \mathrm{day})$ but rather with the total cumulative dose $[1,4,8,9]$. The mechanisms of colistin-induced renal toxicity are unknown, but some investigators have hypothesized that colistin induces membrane permeability and subsequent increases in intracellular cations, anions and water, resulting in cell lysis. Tubular toxicity was suggested by microscopic analysis of urine samples and the time course of renal recovery $[1,4,8,9]$. This tubular toxicity will then evolve towards a form of acute tubular necrosis, which is most of the time reversible. Acute cortical necrosis occurs very seldom. Overall, the incidence of AKI induced by colistin has been overestimated up to now $[1,4,8,9]$.

\section{Colistin Handling during CRRT and Its Relation to Efficacy and Toxicity}

Colistin is predominantly nonrenally cleared in patients with normal kidney function [10]. Colistin undergoes extensive (up to $80 \%$ ) tubular reabsorption. As a consequence, most of the filtered colistin is retained in 
the body and only an extremely low fraction is excreted unchanged in the urine. It can be predicted from recent PK models that a high loading dose is paramount to obtain a rapid bacterial killing effect. Without such a loading dose, steady-state bactericidal colistin concentrations are only reached after $24-48 \mathrm{~h}$ [11].

Very few data exist regarding colistin treatment under conditions of renal replacement. Elimination of colistin has been assessed during intermittent [12] as well as continuous [13-15] dialysis. However, these studies do not provide clinically relevant PK/PD information because colistin doses were either in a toxic [12] or a rather low [13-15] range. Colistin handling is dramatically altered during convective CRRT. In fact, no carrier-mediated mechanism returning colistin from the ultrafiltrate to the blood is present, which virtually excludes reabsorption [10]. Also, the AUC:MIC ratio has been identified as the PK/PD parameter best associated with colistin efficacy [8]. To maximize this ratio, higher doses of colistin may be warranted in the ICU setting. For these reasons, a substantially higher maintenance dose than in patients without AKI (4.5 million IU t.i.d. instead of b.i.d.) should be given during CRRT $[17,18]$. Toxicity risk depends upon either drug accumulation by reabsorption or the presence of too high peak concentrations. During CRRT, continuous loss by filtration permits to avoid dose accumulation of colistin whilst bulk membrane adsorption avoids occurrence of huge or persisting peak concentrations [10, 17]. This explains the absence of colistin toxicity during CRRT even when the aforementioned high doses are administered for a long time period. Still, caution is needed since not all currently used membranes have similar adsorptive capacity [19].

\section{CRRT-Related Strategies to Avoid Colistin Toxicity}

\section{Dose and Modality}

Based on convective elimination under regional isonatremic citrate anticoagulation, continuous venovenous hemofiltration $(\mathrm{CVVH})$ is the preferred CRRT modality in ICU patients. Continuous venovenous hemodiafiltration has not shown any superiority above CVVH for eliminating small molecules [20]. The current KDIGO guidelines recommend a standard dialysis dose of $35 \mathrm{ml} /$ $\mathrm{kg} / \mathrm{h}$ to ascertain delivery of at least $25 \mathrm{ml} / \mathrm{kg} / \mathrm{h}$ [21]. High-volume CVVH does not offer any more benefit [22, 23].

CRRT-Related Strategies to Avoid Colistin Toxicity

\section{Type of Membrane}

Membranes with no or poor adsorptive capacity are not suitable for antibiotic clearance. Drug elimination in CRRT devices equipped with this type of membranes is entirely determined by convection $[13,14]$. In contrast, the novel AN69 ST (acrylonitrile 69 surface treated) membrane allows much higher clearance through adsorption. Elimination occurs in part at the (rapidly saturated) membrane surface but predominantly in its (less easily saturated) bulk [19]. Early saturation is unlikely and frequent membrane changes (e.g. every 12-24 h) are not necessary $[17,19]$. This is corroborated by our experience in a small cohort of patients who supported colistin doses as high as 4.5 million IU t.i.d. for more than 5 consecutive days without exhibiting any side effects or toxicity while the membrane was not changed during this time period $[9,17]$. Polymethyl methacrylate membranes may be capable to adsorb similar amounts of colistin into their bulk [19], but studies reporting on colistin elimination using these particular membranes are lacking. Thus, high colistin doses can only be safely applied when highly adsorptive filters (such as the AN69 ST membrane) are employed. Monitoring of transmembrane pressure and hemopermeability index permit continuous assessment of functional capacity of the dialysis membrane.

\section{Anticoagulant Therapy}

Citrate is the anticoagulant of choice because it effectively combats the clogging process thereby guaranteeing long-term optimal porosity $[24,25]$ and - when using the AN69 ST filter - adsorptive capacity of the membrane [17].

\section{Agenda for Futures Trials on Colistin during CRRT}

While we have summarized the available data on colistin during CRRT in order to provide clinicians with better tools to combat effectively emerging multidrug-resistant Gram-negative infections, we have to remain cautious about several items that would need further validation. This validation should occur in large prospective $\mathrm{PK} / \mathrm{PD}$ studies using serial colistin measurements, which are obviously cumbersome to realize but nevertheless indispensable. Whereas it makes a lot of sense using higher doses of colistin during CRRT (4.5 million IU t.i.d.), we do not have a large PK/PD study (although suggested by some case reports $[9,17])$ that could certify that this dose is really the best one in terms of the AUC:MIC ratio. Besides, as also suggested by some case reports $[9,17]$, we 
do need better data from a large prospective study in order to further confirm or reject the adsorption elimination as dominant mechanism for colistin depuration through CRRT. Along these lines, further refinements of the ideal mode, membrane, frequency of membrane change as well ideal anticoagulation regimen need to be realized accordingly. Regarding the anticoagulation regimen, some case reports $[9,17]$ suggested citrate to be the best choice in case of colistin to enhance elimination, but further confirmation by adequate trials is required, too.

\section{Adsorption of Colistin: An Efficient Shield against Toxicity but Perhaps at the Cost of a Lower AUC:MIC Ratio}

Indeed, adsorption as the main mechanism of elimination using highly adsorptive filters may protect effectively from neuro- and nephrotoxicity but at the cost of lowering perhaps the AUC:MIC ratio despite the high load- ing dose used (9 million IU). Again, better PK/PD data should provide more refined information on the use of these highly adsorptive filters in order to allow for a higher AUC:MIC ratio without putting the patient at risk of greater toxicity.

\section{Conclusions}

Colistin is an intrinsically nephrotoxic and potentially neurotoxic antibiotic. Therapeutic drug monitoring to control its administration is not available in daily ICU practice. Despite these 'drawbacks', patients undergoing CVVH can be treated with long-term colistin therapy at doses up to 4.5 million IU t.i.d. without toxicity. Implementation of such a treatment protocol strictly requires the use of filters allowing high colistin adsorption in the bulk of the membrane in association with citrate anticoagulation for the prevention of early membrane clogging in order to maintain high convection elimination as well.

\section{References}

-1 Spapen HD, Jacobs R, Van Gorp V, Troubleyn J, Honore PM: Renal and neurological side effects of colistin in critically ill patients. Ann Intensive Care 2011;1:14.

$>2$ Bergen PJ, Li J, Rayner CR, Nation RL: Colistin methanesulfonate is an inactive prodrug of colistin against $P$ seudomonas aeruginosa. Antimicrob Agents Chemother 2006;50:1953-1958.

-3 Couet W, Grégoire N, Marchand S, Mimoz O: Colistin pharmacokinetics: the fog is lifting. Clin Microbiol Infect 2012;18:30-39.

4 Yahav D, Farbman L, Leibovici L, Paul M: Colistin: new lessons on an old antibiotic. Clin Microbiol Infect 2012;18:18-29.

$>5$ Deryke CA, Crawford AJ, Uddin N, Wallace MR: Colistin dosing and nephrotoxicity in a large community teaching hospital. Antimicrob Agents Chemother 2010;54:4503-4505.

-6 Hartzell JD, Neff R, Ake J, Howard R, Olson $\mathrm{S}$, Paolino K, et al: Nephrotoxicity associated with intravenous colistin (colistimethate sodium) treatment at a tertiary care medical center. Clin Infect Dis 2009;48:1724-1728.

-7 Kalin G, Alp E, Coskun R, Demiraslan H, Gündogan K, Doganay M: Use of high-dose IV and aerosolized colistin for the treatment of multidrug-resistant Acinetobacter baumannii ventilator-associated pneumonia: do we really need this treatment? J Infect Chemother 2012;18:872-877.

-8 Michalopoulos AS, Falagas ME: Colistin: recent data on pharmacodynamics properties and clinical efficacy in critically ill patients. Ann Intensive Care 2011;1:30.
>9 Spapen HD, Honore PM, Gregoire N, Gobin P, de Regt J, Martens GA, Pierard D, Couet W: Convulsions and apnoea in a patient infected with New Delhi metallo- $\beta$-lactamase- 1 Escherichia coli treated with colistin. J Infect 2011; 63:468-470.

10 Li J, Nation RL, Turnidge JD, Milne RW, Coulthard K, Rayner CR, Paterson DL: Colistin: the re-emerging antibiotic for multidrugresistant Gram-negative bacterial infections. Lancet Infect Dis 2006;6:589-601.

11 Mohamed AF, Karaiskos I, Plachouras D, Karvanen M, Pontikis K, Jansson B, Papadomichelakis E, Antoniadou A, Giamarellou H, Armaganidis A, Cars O, Friberg LE: Application of a loading dose of colistin methanesulfonate in critically ill patients: population pharmacokinetics, protein binding, and prediction of bacterial kill. Antimicrob Agents Chemother 2012;56:4241-4249.

12 Marchand S, Frat JP, Petitpas F, Lemaître F, Gobin P, Robert R, Mimoz O, Couet W: Removal of colistin during intermittent haemodialysis in two critically ill patients. J Antimicrob Chemother 2010;65:1836-1837.

13 Markou N, Fousteri M, Markantonis SL, Zidianakis B, Hroni D, Boutzouka E, Baltopoulos G: Colistin pharmacokinetics in intensive care unit patients on continuous venovenous haemodiafiltration: an observational study. J Antimicrob Chemother 2012;67: 2459-2462.
>14 Cho YS, Yim H, Yang HT, Hur J, Chun W, Kim JH, Lee BC, Seo DK, Park JM, Kim D: Use of parenteral colistin for the treatment of multiresistant Gram-negative organisms in major burn patients in South Korea. Infection 2012;40:27-33.

15 Li J, Rayner CR, Nation RL, Deans R, Boots R, Widdecombe N, Douglas A, Lipman J: Pharmacokinetics of colistin methanesulfonate and colistin in a critically ill patient receiving continuous venovenous hemodiafiltration. Antimicrob Agents Chemother 2005;49: 4814-4815.

16 Plachouras D, Karvanen M, Friberg LE, Papadomichelakis E, Antoniadou A, Tsangaris I, Karaiskos I, Poulakou G, Kontopidou F, Armaganidis A, Cars O, Giamarellou H: Population pharmacokinetic analysis of colistin methanesulfonate and colistin after intravenous administration in critically ill patients with infections caused by gram-negative bacteria. Antimicrob Agents Chemother 2009;53:3430-3436.

17 Honore PM, Jacobs R, Lochy S, De Waele E, Van Gorp V, De Regt J, Martens G, JoannesBoyau O, Boer W, Spapen HD: Acute respiratory muscle weakness and apnea in a critically ill patient induced by colistin neurotoxicity: key potential role of hemoadsorption elimination during continuous venovenous hemofiltration. Int J Nephrol Renovasc Dis 2013;6:107-111.

$>18$ Lee J, Han S, Jeon S, Hong T, Song W, Woo H, Yim DS: Population pharmacokinetic analysis of colistin in burn patients. Antimicrob Agents Chemother 2013;57:2141-2146. 
19 Honore PM, Jacobs R, Joannes-Boyau O, De Regt J, De Waele E, van Gorp V, Boer W, Verfaillie L, Spapen HD: Newly designed CRRT membranes for sepsis and SIRS - a pragmatic approach for bedside intensivists summarizing the more recent advances: a systematic structured review. ASAIO J 2013; 59:99-106.

20 Morimatsu H, Uchino S, Bellomo R, Ronco C: Continuous renal replacement therapy: does technique influence electrolyte and bicarbonate control? Int J Artif Organs 2003;4:289-296.
21 Kellum JA, Lameire N; KDIGO AKI Guideline Work Group: Diagnosis, evaluation, and management of acute kidney injury: a KDIGO summary (part 1). Crit Care 2013; $17: 204$.

22 Joannes-Boyau O, Honoré PM, Perez P, et al: High-volume versus standard-volume haemofiltration for septic shock patients with acute kidney injury (IVOIRE study): a multicentre randomized controlled trial. Intensive Care Med 2013;39:1535-1546

23 Zhang P, Yang Y, Lv R, Zhang Y, Xie W, Chen $\mathrm{J}$ : Effect of the intensity of continuous renal replacement therapy in patients with sepsis and acute kidney injury: a single-center randomized clinical trial. Nephrol Dial Transplant 2012;27:967-973.
24 Honore PM, Joannes-Boyau O, Collin V, Boer W, Gressens B, Janvier G: Practical daily management of extra-renal continuous removal. Reanimation 2008; 17:472-478.

-25 Hofbauer R, Moser D, Frass M, Oberbauer R, Kaye AD, Wagner O, Kapiotis S, Druml W: Effect of anticoagulation on blood membrane interactions during hemodialysis. Kidney Int 1999;56:1578-1583. 